\title{
Liver Function and Bleeding Complications Associated with Lenvatinib
}

\author{
Katsutoshi Ishihara
}

Department of Radiology, Yuai Memorial Hospital, Koga, Japan

Address for correspondence: Katsutoshi Ishihara Department of Radiology, Yuai Memorial Hospital 707 Higashi-Ushigaya, Koga city, Ibaraki, Japan kishihara@yuai-hosp-jp.org

Received: 19.03.2021

Accepted: 22.03.2021
Hepatocellular carcinoma ranks third as the cause of cancer-related death worldwide. Lenvatinib is the novel agent as the first-line treatment for unresectable HCC. Lenvatinib is a multi-tyrosine kinase inhibitor that inhibits the vascular endothelial growth factor receptor (VEGFR 1-3), fibroblast growth factor receptor (FGFR 1-4), plateletderived growth factor receptor a (PDGFRa), stem cell factor receptor (KIT), and rearranged during transfection (RET) [1]. These receptors are important for tumour angiogenesis, and lenvatinib inhibits tumour angiogenesis by inhibiting their function. Moreover, as FGFR, RET, PDGFRa, and KIT play a role in cancer cell proliferation, it is anticipated that lenvatinib directly inhibits the proliferation of cancer cells. In the REFLECT trial, lenvatinib showed noninferiority to sorafenib, with a median overall survival (OS) of 13.6 versus 12.3 months. Additionally, lenvatinib demonstrated noninferiority to sorafenib against HCC and was associated with a significantly higher progression free survival (7.4 vs. 3.7 months; $\mathrm{p}<0.00001$ ), time to progression ( 8.9 vs. 3.7 months $\mathrm{p}<0.0001$ ), and overall response rate $(24.1 \%$ vs. $9.2 \%$; $\mathrm{p}<0.00001$ ) than that of sorafenib [2]. Moreover, lenvatinib was associated with a higher overall response rate according to the modified Response Evaluation
Criteria in Solid Tumors (mRECIST) criteria, and with a higher overall response rate (from $24.1 \%$ in the investigator review to $40.6 \%$ in masked independent review) than sorafenib (from $9.2 \%$ in thr investigator review to $12.4 \%$ in the masked independent review). Lenvatinib became available as a first-line treatment for unresctable HCC in 2018. On the other hand, there are some issues with lenvatinib. In this article, we focused on liver function and bleeding complications associated with lenvatinib administration.

In the REFLECT trial, all the eligible patients had adequate liver function (albumin $\geq 2.8 \mathrm{~g} / \mathrm{dL}$, bilirubin $\leq 3.0 \mathrm{mg} / \mathrm{dL}$, and levels of aspartate aminotransferase, alkaline phosphatase, and alanine aminotransferase were $\leq$ five times the upper limit of normal), and adequate bone marrow function (haemoglobin $\geq$ $8.5 \mathrm{~g} / \mathrm{dL}$, platelet count $\geq 75 \times 10^{9} / \mathrm{L}$, and absolute neutrophil count $\left.\geq 1.5 \times 10^{9} / \mathrm{L}\right)$. Patients with main portal vein invasion, biliary invasion, and tumour burden of more than $50 \%$ of the liver were excluded. Of the total number of eligible patients in the REFLECT trial, 99\% had Child-Pugh class A liver function.

Patients with unresectable HCC are frequently associated with moderate or severe impaired liver function (Child-Pugh class B or class $\mathrm{C}$ hepatic impairment). Therefore, it is of clinical interest to clarify whether lenvatinib can be used in patients with moderate or severe impaired liver function.

Sorafenib, like lenvatinib, is a multikinase inhibitor that acts of VEGF. A cohort study on sorafenib revealed that patients with Child-Pugh class B liver function impairment have lower OS benefits from sorafenib than patients with ChildPugh class A liver function [3]. A phase 2 trial on sorafenib revealed that patients with Child-Pugh class B liver function impairment developed more severe liver toxicities, including hyperbilirubinemia ascites, and encephalopathy, compared to Child-Pugh class A liver function [4]. Similar results have been reported in cases of using lenvatinib. Maruta et al. [5] demonstrated that Child-Pugh class B patients observed high frequency rates of liver function-related adverse events (e.g., elevated aspartate transaminase, hepatic encephalopathy, and bilirubin elevation) due to lenvatinib.

Although a small number of cases were considered in the retrospective study conducted by Komatsu et al. [6], there were no significant differences in the pharmacokinetics, safety, and tolerability of lenvatininb between patients with Child-Pugh class B liver function impairment and patients with Child-Pugh class A liver function [6]. 
In this issue of the Journal of Gastrointestinal and Liver Diseases, the retrospective study by Cosma et al. [7] revealed that the tolerability and toxicity of lenvatinib were similar between patients with Child-Pugh class A liver function and patients with Child-Pugh class B liver function impairment. The study reported the same results as those obtained by Komatsu et al. [6]. The study also reported that patients with Child-Pugh class A liver function have better survival benefits than patients with Child-Pugh class B and class $C$ liver function impairment. It was reported that patients with a Child-Pugh score of 5 points had remarkable higher dose intensity rather than patients with a Child-Pugh score of 6 points or higher [5]. Further investigations are required to adjust the dose of lenvatinib to liver function. Therefore, there has been no evidence that lenvatinib could be recommended for patients with Child-Pugh class B liver function impairment and it may be appropriate to consider lenvatinib for patients only with Child-Pugh class A liver function under the existent circumstances. Moreover, lenvatinib may be contraindicated in patients with Child-Pugh class $\mathrm{C}$ liver function impairment owing to the limited life expectancy and low magnitude of benefit in this population.

The study conducted by Cosma et al. [7] reported that the differences between the Child-Pugh scores at the beginning and end of therapy were not statistically significant. However, Hatanaka et al. [8] reported that the liver function deteriorated from Child-Pugh class A to class B in 43 of the 110 patients (39.1\%) following the administration of lenvatinib. Moreover, Hiraoka et al. [9] reported that patients with Child-Pugh class A had deteriorated to Child-Pugh class B or C liver impairment with $23.4 \%$ of patients after 4 weeks and $23.7 \%$ of patients after 12 weeks of starting lenvatinib treatment. It appears that cumulative data from further cases are necessary because patients who deteriorate from Child-Pugh class A to class B or class $\mathrm{C}$ might have less survival benefits than patients who do not show deterioration. At least, changes in liver function should be noted during lenvatinib administration.

The most common treatment-emergent adverse events among patients who received lenvatinib included hypertension, diarrhea, decreased appetite, and loss of weight. In addition to this, treatment with multikinase inhibitors is associated with the occurrence of haemorrhagic events. The occurrence of haemorrhagic complications with the use of lenvatinib has also been documented. Cerebral hemorrhage was observed in three out of 462 patients in the REFLECT trial [2]. Another phase II trial reported one fatality owing to a ruptured liver tumour within 30 days of receiving the last dose of lenvatinib [10]. In another study, haemorrhagic events occurred in 35\% of patients treated with lenvatinib versus $18 \%$ of patients who received a placebo against thyroid cancer [11]. These phenomena could be attributed to the fact that lenvatinib is a VEGF inhibitor. Intratumoural haemorrhage and rupture of HCCs due to lenvatinib have also been reported [12].

The cause underlying the rupture of HCCs in the liver is unclear, but several hypotheses have been proposed. According to the small room hypothesis, the susceptibility to rupture is thought to be related to the localisation of the tumour in the liver. Li et al. [13] reported that the majority of ruptured HCCs are localised in the left lateral segments (II and III) and right posterior-inferior segment (VI) [13]. These segments have a relatively small room for a space-occupying lesion, compared to the other segments. Therefore, when a tumour grows beyond the available space, the inner pressure splits open the surrounding parenchyma and tears the capsule leading to rupture. According to the vascular injury hypothesis, the increased expression of collagenase, proliferation of elastin, and degradation of type IV collagen fibrils have been observed in small arteries and biopsy specimens from ruptured HCCs [14]. These alterations can cause the small arteries supplying the tumour to turn stiff and brittle, which rupture easily. According to the venous congestion hypothesis, the invasion and occlusion of the hepatic vein leads to intratumoural haemorrhage and increases the intratumoural pressure, leading to rupture [15]. Necrosis within the tumour or rapid tumour growth can also lead to increased intratumoural pressure and consequent rupture. The antitumoural effect of lenvatinib may also cause HCCs to rupture. As lenvatinib inhibits tumour angiogenesis, the remaining poorly developed tumour microvasculature tends to collapse.

In the previous issue of the Journal of Gastrointestinal and Liver Diseases, Higashino et al. [16] described a case of spontaneous rupture of lung metastasis from HCC after the initiation of lenvatinib therapy. Bleeding from lung metastasis due to HCC is very rare. A study reported only 21 cases of haemothorax due to thoracic metastasis or direct invasion of HCC into the chest [17]. With the exception of the present case, there have not been case reports of rupture of lung metastases due to HCCs during the administration of lenvatinib in PubMed.

The susceptibility of HCCs to rupture is thought to be related to their hepatic localisation; however, it is difficult to assume that tumour location in the lung will produce any difference. This is because it is difficult to identify a segment that occupies a small volume in the lung tissue. Vascular injuries and venous congestions can occur during the rupture of lung metastases. In addition, the adverse events of lenvatinib may be observed, owing to the inhibition of VEGF.

Uchida-Kobayashi et al. [12] reported that five cases of the 68 patients in their retrospective study developed tumour haemorrhage, including tumour rupture and intratumoural haemorrhage. The average dosing periods of lenvatinib was only $4.4 \pm 2.2$ days in all the five cases. It was additionally observed that patients with bleeding had larger tumours (maximum tumour diameter of $97.5 \pm 46.4 \mathrm{~mm}$ ) than patients without bleeding (maximum tumour diameter of $38.2 \pm 28.8$ $\mathrm{mm})$.

The rupture of lung metastasis from HCC occurred after seven days of lenvatinib administration [16]. Tumour rupture caused by lenvatinib may tend to occur within a relatively early period of administration. It is therefore necessary to accumulate more data from further cases in the future. In this case, a large lung metastasis was observed. It is necessary to consider the risk of bleeding prior to the initiation of lenvatinib for large tumours.

In conclusion, the deterioration of liver function needs to be considered when using lenvatinib. It may be appropriate to consider lenvatinib for patients with Child-Pugh class A liver function and administration of lenvatinib is not recommended 
for patients with Child-Pugh class B liver function impairment under the existent circumstance. It is also necessary to consider the risk of tumour rupture in the early period after starting lenvatinib in large HCCs including metastases.

\section{Conflicts of interest: None to declare}

Acknowledgements: I would like to thank Editage (www.editage. com) for the English language editing.

\section{REFERENCES}

1. Suyama K, Iwase H. Lenvatinib: A Promising Molecular Targeted Agent for Multiple Cancers. Cancer Control 2018;25:1073274818789361. doi:10.1177/1073274818789361

2. Kudo M, Finn RS, Qin S, et al. Lenvatinib versus sorafenib in first-line treatment of patients with unresectable hepatocellular carcinoma: a randomised phase 3 non-inferiority trial. Lancet 2018;391:1163-1173. doi:10.1016/S0140-6736(18)30207-1

3. Pinter M, Sieghart W, Graziadei I, et al. Sorafenib in unresectable hepatocellular carcinoma from mild to advanced stage liver cirrhosis. Oncologist 2009;14:70-76. doi:10.1634/theoncologist.2008-0191

4. Abou-Alfa GK, Schwartz L, Ricci S, et al. Phase II study of sorafenib in patients with advanced hepatocellular carcinoma. J Clin Oncol 2006;24:4293-4300. doi:10.1200/JCO.2005.01.3441

5. Maruta S, Ogasawara S, Ooka Y, et al. Potential of lenvatinib for an expanded indication from the REFLECT trial in patients with advanced hepatocellular carcinoma. Liver Cancer 2020;9:382-396. doi: $10.1159 / 000507022$

6. Komatsu S, Yano Y, Sofue K, et al. Assessment of lenvatinib treatment for unresectable hepatocellular carcinoma with liver cirrhosis. HPB (Oxford) 2020;22:1450-1456. doi:10.1016/j.hpb.2020.03.002

7. Cosma LS, Weigand K, Müller_Schilling M, Kandulski A. Lenvatinib as first-line treatment of hepatocellular carcinoma in patients with impaired liver function in advanced liver cirrhosis: real world data and experience of a tertiary hepatobiliary center. J Gastrointestin Liver Dis 2021;30:247-253. doi:10.15403/jgld-3345

8. Hatanaka T, Kakizaki S, Nagashima T, et al. Liver function changes in patients with hepatocellular carcinoma treated with lenvatinib: predictive factors of progression to Child-Pugh class B, the formation of ascites and the candidates for the post-progression treatment. Cancers (Basel) 2020;12:2906. doi:10.3390/cancers12102906

9. Hiraoka A, Kumada T, Kariyama K, et al. Clinical features of lenvatinib for unresectable hepatocellular carcinoma in real-world conditions: Multicenter analysis. Cancer Med 2019;8:137-146. doi:10.1002/cam4.1909

10. Ikeda K, Kudo M, Kawazoe S, et al. Phase 2 study of lenvatinib in patients with advanced hepatocellular carcinoma. J Gastroenterol 2017;52:512-519. doi:10.1007/s00535-016-1263-4

11. Nair A, Lemery SJ, Yang J, et al. FDA Approval Summary: Lenvatinib for Progressive, Radio-iodine-Refractory Differentiated Thyroid Cancer. Clin Cancer Res 2015;21:5205-5208. doi:10.1158/1078-0432.CCR-15-1377

12. Uchida-Kobayashi S, Kageyama K, Yamamoto A, et al. Lenvatinibinduced tumour-related hemorrhages in patients with large hepatocellular carcinomas. Oncology 2021;99:186-191. doi:10.1159/000510911

13. Li J, Huang L, Liu CF, et al. Risk factors and surgical outcomes for spontaneous rupture of BCLC stages A and B hepatocellular carcinoma: a case-control study. World J Gastroenterol 2014;20:9121-9127.

14. Zhu LX, Geng XP, Fan ST. Spontaneous rupture of hepatocellular carcinoma and vascular injury. Arch Surg 2001;136:682-687. doi:10.1001/archsurg.136.6.682

15. Zhu LX, Wang GS, Fan ST. Spontaneous rupture of hepatocellular carcinoma. Br J Surg. 1996;83:602-607. doi:10.1002/bjs.1800830507

16. Higashino M, Sugiura R, Yamamoto Y, Naruse H, Sakamoto N. A spontaneous rupture of lung metastasis from hepatocellular carcinoma after introduction of lenvatinib. J Gastrointestin Liver Dis 2021;30:169170. doi:10.15403/jgld-3025

17. Yen CW, Hsu LS, Chen CW, Lin WH. Hepatocellular carcinoma with thoracic metastases presenting as hemothorax: A case report and literature review. Medicine (Baltimore) 2018;97:e10945. doi:10.1097/ MD.0000000000010945 